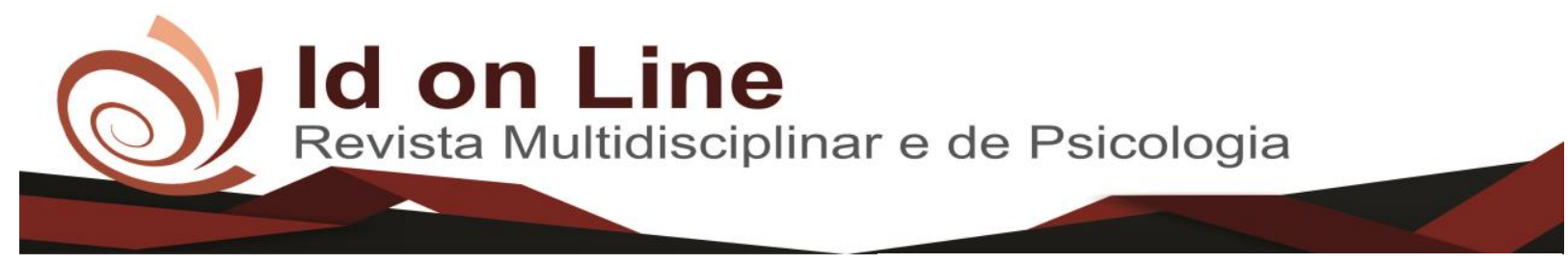

Artigo

\title{
Saúde do Homem na Atenção Básica: Prevenção e Participação nos Programas
}

\author{
Gilciéle Novais Rocha ${ }^{1}$; Isadora Ferreira Araújo ${ }^{2 ;}$ Júlia Sousa Santos Nunes $^{3}$
}

\begin{abstract}
Resumo: O público masculino apresenta resistência em relação à procura pelas Unidades de Saúde, o que o torna mais susceptível ao adoecimento/falha na prevenção. Esta pesquisa foi desenvolvida com o propósito de avaliar a participação do público masculino nos programas de saúde em uma Unidade Básica do município de Vitória da Conquista na Bahia, cuja característica metodológica foi a de uma pesquisa de campo, de caráter quantitativo, exploratória-descritiva. Foi aplicado um questionário aos usuários da Unidade em questão onde foram entrevistados 47 homens, tendo como maioria na faixa etária de 60 anos ou mais $(46,8 \%)$, seguida de 50 a 59 anos $(36,2 \%)$. Quanto ao grau de escolaridade 33 participantes $(70,2 \%)$, ou seja, o maior número estudou até o ensino fundamental. Observou-se na pesquisa que os participantes apresentam doença cardiovascular, fazem uso do álcool socialmente e do tabaco, embora mais de $70 \%$ dos entrevistados realizam alguma atividade física, o que demonstra que existe a ciência da prevenção, apesar da persistência de alguns hábitos prejudiciais a saúde. Os dados mostram que o público masculino procura mais os serviços de saúde em busca do tratamento ao invés da prevenção, o que pode evidenciar uma maior preocupação pela saúde quando esta se encontra afetada.
\end{abstract}

Palavras-Chave: Saúde do Homem, Prevenção Primária, Centros de Saúde.

\section{Man's Health nn Basic Attention: Program Prevention and Participation}

\begin{abstract}
The male public is resistant to the search for health units, which makes them more susceptible to illness / failure in prevention. This research was developed with the purpose of evaluating male participation in health programs in a Basic Unit in the city of Vitória da Conquista in Bahia, whose methodological characteristic was that of a field research, of a quantitative, exploratory-descriptive character. A questionnaire was applied to the users of the Unit in question where 47 men were interviewed, with a majority in the age group of 60 years or more (46.8\%), followed by 50 to 59 years (36.2\%). Regarding the degree of schooling 33 participants $(70.2 \%)$, that is, the largest number studied until elementary school. It was observed in the research that the participants present cardiovascular disease, use alcohol and tobacco, although more than $70 \%$ of those interviewed regularly perform physical activity, which shows that there is a science of prevention, but some habits persist. The data show that the male public seek health services more in search of treatment rather than prevention, which may indicate a greater concern for the health of this population.
\end{abstract}

Keywords: Men's Health, Primary Prevention, Health Centers.

\footnotetext{
${ }^{1}$ Graduada em enfermagem. Faculdade Independente do Nordeste - FAINOR. Vitoria da Conquista, BA, Brasil. Email: gil.mombassa@hotmail.com;

${ }^{2}$ Coorientadora. Enfermeira no Centro de Reabilitação de Vitória da Conquista, BA, Brasil. Especializada em Gestão em Saúde, em Estomaterapia e em Saúde Coletiva. Email: isadoraferreiraaraujo@ hotmail.com;

${ }^{3}$ Prof ${ }^{a}$.de Enfermagem e Msc. em Saúde Coletiva. Faculdade Independente do Nordeste- FAINOR. Vitoria da Conquista, BA, Brasil. Email: juliassenf@yahoo.com.br.
} 


\section{Introdução}

Os homens em geral só vão em busca de atendimento médico quando são acometidos por alguma enfermidade grave, o que gera uma importante preocupação com a saúde masculina pelos serviços de saúde. Tal situação é tão frequente que possui uma tendência cultural, pois muitos destes indivíduos acreditam que o diagnóstico de uma doença está relacionado à fraqueza, e este não quer apresentar nenhum tipo de fragilidade (VIEIRA, 2013).

A população masculina além de ver as unidades de saúde como um espaço feminilizado. Observa-se que nos serviços de saúde as campanhas voltadas para saúde do homem ocorrem com menor frequência, geralmente nos meses destinados para prevenção do câncer de próstata e pênis, ou ações voltadas para conscientizar sobre o uso e abuso de álcool e outras drogas que são mais constantes em pessoas do gênero masculino (VIEIRA, 2013).

No Brasil, nas últimas duas décadas aumentou o número de estudos sobre saúde masculina, sendo que a relação homem e saúde é objeto de atenção nos meios acadêmicos e no contexto dos serviços. Incluir a participação do homem nas ações de saúde é, no mínimo, um desafio (SCHRAIBER, 2005). É fundamental que se promova ações de saúde que contribuirá para a compreensão da realidade atual masculina nos seus diversos aspectos, de forma a melhorar a qualidade de vida do homem (JULIÃO, 2011).

A inclusão do homem nos programas de saúde é um desafio muito grande, sendo que as principais dificuldades estão em não admitir e aceitar suas fragilidades, e não prezar a importância do cuidado com a saúde. Alguns não procuram os serviços devido à incompatibilidade de horário com a jornada laboral, que dificulta o acesso (MACHIM, 2011).

Assim sendo são relevantes estudos direcionados a população masculina na busca da prevenção nas unidades de Atenção Primária a Saúde nas Unidades Básicas.

Em 2008, o Ministério da Saúde constituiu a Política Nacional de Atenção Integral à Saúde do Homem (PNAISH), com o objetivo de promover a melhorias nas condições de saúde da população masculina brasileira, colaborando, de modo ativo para a redução da morbidade e mortalidade por meio das dificuldades racionais dos fatores de risco e mediante a facilitação ao acesso, às ações e aos serviços de assistência total à saúde. Promovendo ações de saúde que contribuíam consideravelmente para a percepção da realidade do homem nos seus vários contextos socioculturais e político-econômicos, está alinhada com a Política Nacional de 
Atenção Básica - porta de entrada do Sistema Único de Saúde -, principalmente com suas estratégias de humanização, na procura pelo fortalecimento das ações e dos serviços disponibilizados para a população (CABACINHA, 2014).

De acordo com o art. $3^{\circ}$ da Política Nacional de Atenção Integral à Saúde do Homem há diretrizes que precisam ser observadas ao elaborar planos, programas, projetos e ações de saúde voltadas à população masculina, que envolve entre outras a integralidade e organização dos serviços públicos de saúde de modo a acolher e fazer com que o homem sinta-se integrado (BRASIL, 2008).

Portanto a Política orienta as ações e serviços de saúde para o homem, com integralidade e equidade, primando pela humanização da atenção. Focaliza a necessidade de mudanças de paradigmas no que compete à percepção da população masculina no que se diz respeito ao cuidado com a sua saúde e de sua família (CABACINHA, 2014).

No que se referem à saúde do homem, algumas patologias ocorrem com mais frequência como, por exemplo, as doenças cardiovasculares, causas externas, neoplasias, doenças do aparelho respiratório, obesidade, alcoolismo e tabagismo (CABACINHA, 2014).

Diante do panorama realizado acima, nota-se que a atenção à saúde do homem tem buscado atender suas demandas, e as morbidades prevalentes na população masculina em sua maioria são passíveis de prevenção e tem os respectivos tratamentos. Logo, podemos visualizar assim, a importância do fortalecimento do vínculo dos homens com as unidades de saúde. É importante também que os serviços públicos de saúde sejam organizados de maneira a fazer com que o homem se sinta integrado e acolhido. A implementação da política precisará ocorrer de modo integral às outras políticas existentes, numa lógica hierarquizada de atenção à saúde, privilegiando a atenção primária como porta de entrada de um sistema de saúde universal, integral e equânime (BRASIL, 2008).

Desse modo, o presente trabalho tem como objetivo avaliar a participação do público masculino na unidade de saúde e a busca pela prevenção ou tratamento. Especificando o perfil dos homens que procuram a UBS e identificar quais os principais motivos que levam à procura dos serviços. 


\section{Método}

A abordagem do estudo foi por pesquisa de campo, escolhida para atingir os objetivos da pesquisa. De natureza quantitativa, e quanto aos objetivos exploratória-descritiva. A pesquisa exploratória proporcionou uma visão ampliada sobre a situação em estudo e suas variáveis, identificando como as variáveis se inter-relacionam (DYNIEWICZ, 2011).

O estudo descritivo tem como objetivos a observação, a descrição e a documentação dos aspectos da situação pesquisada, permitindo ao pesquisador controlar diversas variáveis. Dessa forma, o estudo exploratório descritivo, aliado à abordagem quantitativa, fornece ao pesquisador uma visão ampla sobre a forma através da qual as variáveis se configuram e influenciam determinados acontecimentos, além da exploração condizente com a ideia principal do problema que a pesquisa se propõe revelar (OLIVEIRA, 1999).

O local escolhido para estudo foi a Unidade Básica de Saúde localizada no Bairro Vila América na cidade de Vitória da Conquista - Bahia, onde foram realizadas as atividades de estágio, devido a facilidade de acesso e familiaridade com os profissionais que atuam na instituição. Os participantes voluntários foram 47 homens que buscaram a Unidade de Saúde para atendimento preventivo ou curativo no período de realização do estudo, que ocorreu entre os dias 24 de outubro a 15 de novembro de 2016.

O instrumento que foi utilizado para pesquisa, consistiu em um questionário semiestruturado, composto com questões com foco no objetivo geral, aplicado aos homens que frequentaram a unidade no período da aplicação e consentiram em participar.

As informações colhidas foram armazenadas em planilhas do programa Microsoft Office Excel, lidas e analisada de forma minuciosa, com análise estatística descritiva, identificando as respostas que trouxeram informações percentuais para gerar informação.

O formulário, o termo de consentimento livre e esclarecido, bem como o projeto foram aprovados pelo Comitê de Ética em Pesquisa da Faculdade Independente do Nordeste FAINOR e pelo Comitê de Ética do Polo de Educação Continuada de Vitória da Conquista BA. 


\section{Resultados e Discussão}

Tabela 1 - Dados sociodemográficos de usuários de uma unidade básica de saúde de Vitória da Conquista - BA.

\begin{tabular}{ccc}
\hline & Dados Sociodemográficos dos Participantes \\
\hline Idade & Totais Parciais & $\%$ \\
19 a 39 anos & 04 & 8,5 \\
40 a 49 anos & 04 & 8,5 \\
50 a 59 anos & 17 & 36,2 \\
60 ou mais & $\mathbf{2 2}$ & $\mathbf{4 6 , 8}$ \\
& & \\
Estado Civil & & \\
Solteiro & 09 & 19,1 \\
Casado & $\mathbf{2 0}$ & $\mathbf{4 2 , 6}$ \\
União Estável & 17 & 36,2 \\
Viúvo & 01 & 2,1 \\
& & \\
Escolaridade & & 0,0 \\
Analfabeto & 0 & $\mathbf{7 0 , 2}$ \\
Ensino Fundamental & $\mathbf{3 3}$ & 27,7 \\
Ensino Médio & 13 & 2,1 \\
Ensino Superior & 01 & \\
& & \\
Modalidade de & & $\mathbf{6 1 , 7}$ \\
Trabalho & $\mathbf{2 9}$ & 38,3 \\
Trabalho Formal & 18 & \\
Trabalho Informal & &
\end{tabular}

Fonte: Própria (2016)

A tabela 1 elenca características utilizadas para traçar o perfil socioeconômico da população estudada. Foram entrevistados 47 homens, com maioria dentro da faixa etária de 60 anos ou mais (46,8\%), seguidos pelos adultos de 50 a 59 anos $(36,2 \%)$.

A população idosa participa ativamente das unidades de saúde, devido ao desenvolvimento de doenças crônicas que requer atenção periódica. É importante ressaltar que para um envelhecimento com saúde, é necessário ter uma boa qualidade de vida, com hábitos saudáveis. Dentro da unidade estes podem ser incentivados através de políticas nacionais, como por exemplo, o programa de saúde da família, programa de reabilitação para idosos, no intuito de evitar complicações na idade avançada, como inabilidade, sedentarismo e mutualidade (VERDANA, 2008). A população jovem busca menos as unidades, por possuírem uma jornada de trabalho maior que os mais velhos e também pelo fato de estudarem mais (BROLEZI, 2014). 
Em relação ao estado civil, pode-se notar que a maioria dos participantes são casados ou estão em uma união estável. Pesquisas apontam que homens casados ou em convivência com uma parceira, procuram mais os serviços de saúde do que os solteiros, o porquê dessa afirmação é que estes homens vão em busca de atendimento influenciados pelas suas parceira (SCHRAIBER, 2005).

Quanto ao nível de escolaridade, 33 (70,2\%) dos participantes estudaram até o ensino fundamental. Relacionado à modalidade de trabalho, 29 homens $(61,7 \%)$ possuem trabalho formal e 19 homens $(38,3 \%)$ trabalho informal.

Pelo fato da preocupação masculina, ante a sua função de provedores da sua família, a atividade trabalhista ter um lugar em destaque. Entretanto, em pessoas de baixa condição social e baixo nível escolar, o que reforça o papel historicamente atribuído ao homem de ser responsável pelo sustento da família é maior (ALBANO, 2010).

Tabela 2 - Dados sobre Condições/Situações Gerais de Saúde dos Entrevistados.

\begin{tabular}{lcc}
\hline \multicolumn{3}{c}{ Dados Condições/Situações Gerais de Saúde } \\
\hline $\begin{array}{c}\text { Consumo do Álcool } \\
\text { Consomem }\end{array}$ & Totais Parciais & $\%$ \\
Socialmente & $\mathbf{3 2}$ & $\mathbf{6 8 , 1}$ \\
Não Consomem & 15 & 31,9 \\
Consumo do Tabaco & & \\
$\quad$ Fumante & $\mathbf{2 6}$ & $\mathbf{5 5 , 3}$ \\
Não Fumante & 21 & 44,7 \\
& & \\
Outras Drogas & $\mathbf{0 , 0}$ \\
Hipertensão & & \\
Sim & $\mathbf{3 1}$ & $\mathbf{6 6 , 0}$ \\
Não & 16 & 34,0 \\
Diabetes & & \\
Sim & $\mathbf{2 8}$ & $\mathbf{5 9 , 6}$ \\
Não & 19 & 40,4 \\
& & \\
Doença Cardíaca & $\mathbf{1 2}$ & $\mathbf{2 6 , 6}$ \\
Sim & 35 & $\mathbf{7 4 , 4}$ \\
Não & &
\end{tabular}

Fonte: Própria (2016) 
Foram levantados os questionamentos quanto ao uso do álcool onde 68,1\% (32 usuários), relataram uso de álcool socialmente. O álcool é um grande problema para a população masculina, a prevalência de dependência é maior em indivíduos do sexo masculino. As consequências do seu uso indiscriminado estigmatizam o indivíduo e promove a exclusão social. O alcoolismo quando comparado com outros problemas de saúde, é responsável por gerar três vezes mais licenças médicas, aumenta as chances de acidente do trabalho, entre outras consequências como violência familiar e criminal, dependência física e psíquica (INCA, 2016).

Em relação ao tabaco 55,3\% (26 pessoas) informaram a prática. O tabagismo é considerado a principal causa de morte evitável no mundo. Dados apontam que $63 \%$ das letalidades estão relacionadas às doenças crônicas não transmissíveis. Seu consumo mata milhões de pessoas a cada ano. Sendo assim, se continuar, a partir de 2030 o tabaco matará por volta de 08 milhões de indivíduos anualmente e $80 \%$ dessas mortes ocorrerão nos países menos desenvolvidos (DYNIEWICZ, 2011). Nenhum Dos pesquisados informou o uso de drogas ilícitas. É sabido que, o uso das drogas ilícitas é bem disseminado na sociedade, levando a prejuízos acumulativos à saúde desses usuários (DYNIEWICZ, 2011).

Em relação à hipertensão, 31 participantes $(66,0 \%)$, relataram ser portador da doença crônica, e se referindo ao diabetes mellitus, 28 participantes $(59,6 \%)$ são portadores. A Hipertensão e o Diabetes são as doenças crônicas mais presentes, quando comparadas às doenças cardiovasculares apresentam números superiores na pesquisa realizada. Os fatores de risco para essas doenças são divulgados geralmente pelas redes sociais, TV, serviços de saúde, no entanto, a prevenção não é tida como prioridade por grande parte da população, aumentando ainda mais os casos das doenças e complicações (MACHIN, 2011).

A hipertensão arterial é descrita como uma disfunção clínica multifatorial associada, geralmente às alterações metabólicas e mudanças na estrutura. De acordo com a VI Diretrizes Brasileiras de Hipertensão, o diagnóstico é concluído a partir de altos níveis de pressão arterial, tendo como valores limítrofes 140/90 mmHg em, no mínimo, duas aferições, e daí o paciente segue em acompanhamento e medicação. Quando não controlada, o indivíduo pode sofrer consequências graves, principalmente, o acidente vascular encefálico e uma possível isquemia no coração (SILVA, 2016).

Relacionado ao Diabetes Mellitus, que é entendido como um grupo de distúrbios metabólicos, com condição comum a hiperglicemia. O diabetes é classificado de acordo com 
sua etiologia. O tipo 01 surge quando há uma destruição das células pâncreas, acarretando em falta de insulina no órgão. O tipo 02 é o mais incidente, caracterizado por defeitos na ação e secreção de insulina. Seu diagnóstico é identificado pelo aparecimento de sintomas como, por exemplo: poliúria, polidipsia, perda de peso e glicêmicos em jejum com valor superior a 126 $\mathrm{mg} / \mathrm{dl}$. As complicações que podem vir a acontecer está o infarto agudo do miocárdio, o acidente vascular encefálico, a insuficiência renal crônica, amputação de membros inferiores( o pé diabético é o mais afetado) e a cegueira definitiva (SILVA, 2016).

Às doenças cardíacas 26,6\% (12 participantes), relataram que são portadores da doença. As doenças cardiovasculares estão em primeiro lugar entre as causas de morte no Brasil e consistem em quase um terço dos óbitos, totalizando 300.000 mortes por ano. Diariamente é registrada uma média de 820 óbitos (BROLEZI, 2014).

Gráfico 1 - Distribuição dos participantes quanto a Pratica de Atividade Física. Vitória da Conquista, BA.

$$
\text { - Sim Não }
$$

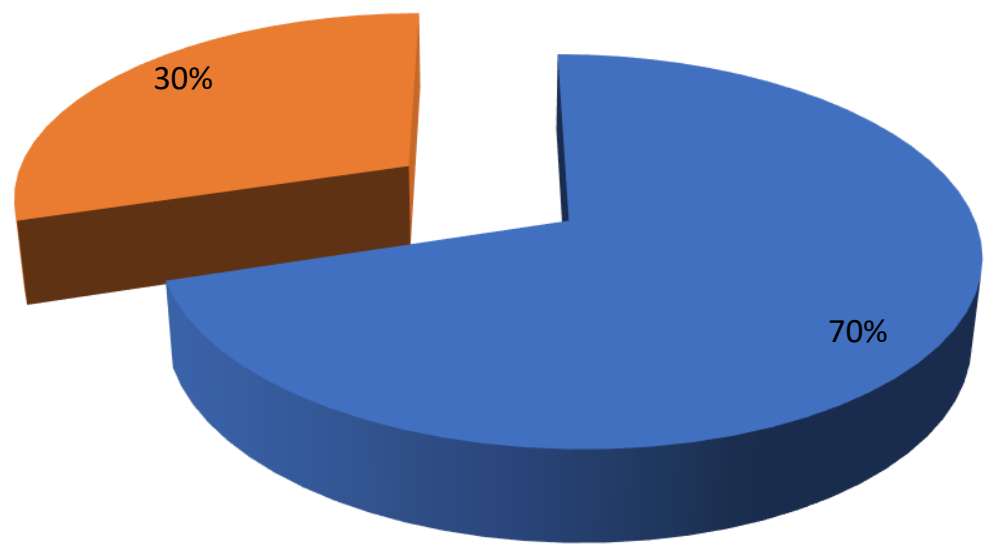

Fonte: Própria (2016)

Em contrapartida, na população pesquisada, quando indagados sobre a prática de atividade física, $33(70 \%)$ dos usuários afirmaram que praticam alguma atividade física. O que demonstra que os homens estão se preocupando com a saúde. 
Estudos anteriores revelaram que mudanças de estilo de vida, com escolha de hábitos de vida mais saudáveis e a redução de comportamentos de risco, podem diminuir as chances de agravos das doenças garantindo assim condições de saúde (MACHIN, 2011).

Diversos são os benefícios da prática de atividade física, chegando a evitar várias doenças, porque a prática é fundamental para controlar a circulação, melhora a pressão arterial porque diminui a pressão sanguínea elevada, fundamental para prevenção de diabetes, diminuem as chances de osteoporose. A prática atua até na saúde psicológica, evitando assim a ansiedade, diminui o estresse, previne a depressão (BRITO, 2011).

A pratica de atividade física é contraindicada para pacientes com estenose aórtica ou pulmonar, a cardiomiopatia hipertrófica, arritmias cardíacas avançadas, a insuficiência cardíaca descompensada, a miocardite e a insuficiência coronária instável. Para pacientes com infecções agudas, a DPOC, o diabetes mellitus e a hipertensão arterial, quando descompensados, a contraindicação pode ser temporária (SILVA, 2016) .

Gráfico 2 - Participação e busca dos usuários entrevistados na Unidade de Saúde. Vitória da Conquista, BA.

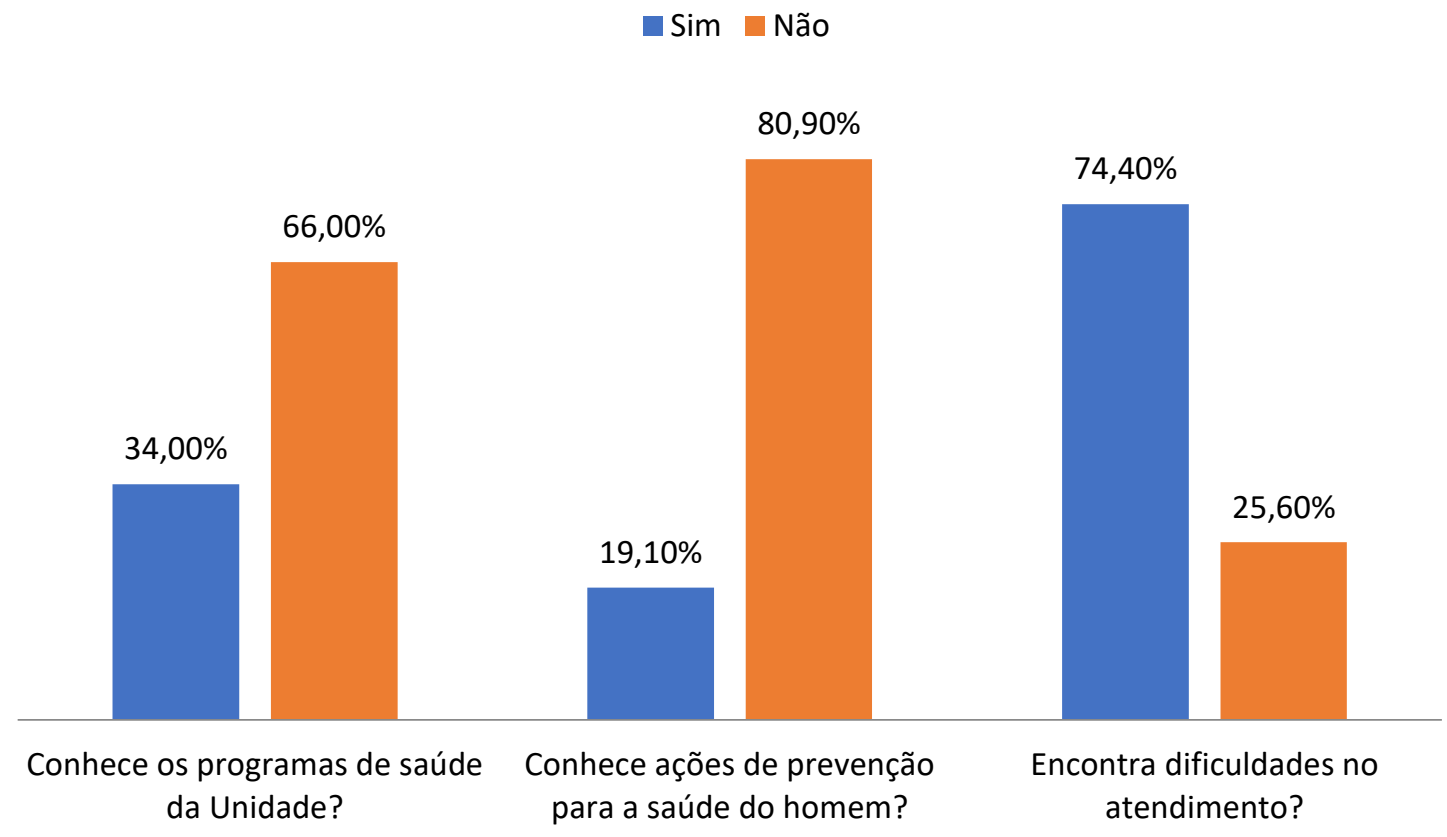

Fonte: Própria (2016) 
O gráfico acima nos mostra um panorama da participação dos usuários nas unidades de saúde. Podemos observar que alguns usuários não estão atualizados acerca das rotinas e dos programas nas unidades de saúde, onde $66 \%$ disseram que não conhecem os programas de saúde da unidade.

E relacionado ao conhecimento das ações de prevenção para a saúde do homem chamou atenção quando 80,90\% (38 usuários) responderam que desconhecem. É considerado baixo o conhecimento da população masculina acerca dos serviços de saúde das unidades.

Pesquisas realizadas nos mostram que os homens alegam o motivo para a não procura pelos serviços é devido à dificuldade de acesso, falta de unidades especificamente voltadas para os serviços do homem, e representação do cuidar como função feminina. Ter que enfrentar filas intermináveis que, muitas vezes, causam a "perda" de um dia inteiro de trabalho, desestimula a procura pelos serviços (MACHIN, 2011 e ALBANO, 2010).

Elencar os motivos que afastam os homens dos serviços de saúde é um desafio, principalmente porque se trata de questões de gênero, cultura, ideia de que a busca por atendimento em saúde é uma demonstração de fragilidade. $\mathrm{O}$ ambiente das unidades que geralmente são mais feminilizados, tanto na presença de paciente quanto dos profissionais e a falta de programas específicos para o público masculino (VIEIRA, 2013).

Foi observado que 74,40\% (34 usuários) encontram dificuldades sendo: 17\% com dificuldade em marcação de exames e escassez de medicamentos, $25 \%$ em dificuldade para marcação de consultas e 32,40\% não quiseram especificar, apenas disseram que sim.

Gráfico 3 - Busca pelos serviços na Unidade foi para prevenção ou tratamento. Vitória da Conquista, BA.

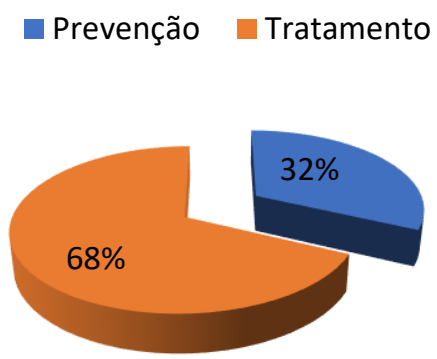

Fonte: Própria (2016) 
No entanto, acerca do motivo de procura à Unidade de Saúde 68\% (32 homens) buscaram a unidade para tratamento e apenas 32\% (15 homens) para prevenção. É fundamental que o homem seja incentivado a buscar as unidades para prevenção, onde terão acesso às atividades educativas, realização de exames de rotina, vacinação, fortalecimento do vínculo. Os dados encontrados conferem com pesquisas realizadas no que se diz respeito em que a maioria dos homens avalia como ter saúde adequada, sem necessidade de busca pela prevenção. Sabendo que muitos agravos poderiam ser evitados se os homens realizassem as medidas preventivas regularmente, o que se observa é que muitos homens tem deficiência em reconhecer suas necessidades. A cada três mortes de cidadãos adultos duas são do sexo masculino, os homens vivem sete anos a menos que as mulheres quando comparado com doenças crônicas e independente dessa taxa alta de morbimortalidade, a maioria dos homens continuam procurando menos as unidades (MACHIN, 2011 e CABACINHA, 2014).

\section{Considerações Finais}

Dos homens entrevistados, a maioria são idosos, casados, ou com relação estável e com escolaridade no ensino fundamental. Observou-se na pesquisa que grande parte dos participantes apresenta alguma doença cardiovascular, fazem uso do álcool socialmente e do tabaco, embora a maioria dos homens entrevistados realizem atividade física regularmente, o que demonstra que existe a ciência da prevenção, mas alguns hábitos persistem. $\mathrm{O}$ desconhecimento e a fragilidade na relação com as unidades de saúde foram reafirmados com a pesquisa. Notou-se que a participação dos homens nas unidades de saúde ainda é pequena, principalmente a população jovem.

Pôde se observar que a maior parte dos usuários vão às unidades de saúde em busca do tratamento, esse dado encontrado significa que os homens não se preocupam tanto com a prevenção, e apenas procuram uma unidade de saúde quando estão com algum sintoma.

Destaca-se ainda a necessidade de um programa específico para a saúde do homem nas Unidades de Saúde, bem como a intensificação da realização de atividades educativas que deem maior empoderamento aos usuários, proporcionando-lhes assim melhor autocuidado e práticas 
saudáveis. Logo as ações e atividades de promoção de saúde para o público masculino, devem ser sempre realizadas, tanto para instruir a população como também para facilitar e ampliar o seu acesso aos serviços de saúde.

\section{Referências}

ALBANO BR, Basílio MC, Neves JB. Desafios para inclusão dos homens nos serviços de atenção primária à saúde. Revista Enfermagem Integrada, Ipatinga: Unileste - MG, v. 3, n. 2, nov/dez, 2010.

ALVES, R.F.; SILVA, R.P.; ERNESTO, M.V; LIMA, A.G.B.; e SOUZA, F.M. Gênero e saúde: o cuidar do homem em debate. Psicologia: teoria e prática, 2011, 13(3), 152-166.

BRASIL Ministério da Saúde. Secretária de Atenção à saúde. Departamento de Ações Programáticas Estratégicas. Política Nacional de Atenção Integral à Saúde do Homem, 2008.

BRASIL Ministério da Saúde. Secretária de Atenção à saúde. Departamento de Ações Programáticas Estratégicas. Política Nacional de Atenção Integral à Saúde do Homem. Princípios e diretrizes. Brasília - DF, Dezembro, 2009.

BRITO R.S.de; ARAUJO, D.dos S.L. Percepção de homens hipertensos e diabéticos sobre a assistência recebida em Unidade Básica de Saúde. Revista Eletrônica de Enfermagem, v. 13, n. 4, 2011.

BROLEZI, E.A.; MARQUES, G.O; MARTINEZ, L.C.B. As principais causas de adoecimento e morte em homens no Brasil. Unifia revista eletrônica saúde foco, 2014.

CABACINHA, R. de O. M; MORAES, C.D.; BARBOSA, A.D.S.; PINHO, H.A.L.de; Condições sociodemográficas e de saúde autorreferidas de homens em uma unidade de saúde. Revista da Rede de Enfermagem do Nordeste - Rev Rene 2014 set-out; 15(5):804-11.

CRUVINEL, T.A.C. Promoção da saúde e qualidade de vida nos idosos na saúde da família. Monografia (Trabalho de Conclusão de Curso)-Universidade Federal de Minas Gerais, Uberaba, 2009.

DYNIEWICZ, A.M. Metodologia da pesquisa em saúde para iniciantes. São Paulo: Difusão, 2011. 207 p.

INCA. Tipos de câncer. Disponível em: <http://www2.inca.gov.br/wps/wcm/connect/tiposdecancer/site/home> Acesso em: 08 de maio, 2016. 
JULIÃO, G.G; e WEIGELT, L.D. Atenção à saúde do homem em unidades de estratégia de saúde da família. Revista de Enfermagem da UFSM, 2011, 1(2), 144-152.

MACHIN, R.; COUTO, M.T.; SILVA, G.S.N.D.; SCHRAIBER, L.B.; GOMES, R,S,; FIGUEREDO, W.D.; \& PINHEIRO, T.F. Concepções de gênero, masculinidade e cuidados em saúde: estudo com profissionais de saúde da atenção primária. Ciência e Saúde Coletiva, 2011.

OLIVEIRA, S.L. de. Tratado de metodologia científica: projetos de pesquisa, TGI, TCC, monografia, dissertações, e teses. Rio de Janeiro: Pioneira, 1999. 320 p.

ROSCANI, M.G. Como diagnosticar e tratar insuficiência cardiaca. Editora Moreira Jr. $10 / 2013$.

SCHRAIBER, L.B.; GOMES, R. e COUTO, M.T. Homens e saúde na pauta da Saúde Coletiva. Rev. Ciênc. Saúde coletiva, 2005, vol. 10, nº 1, Rio de Janeiro.

SILVA, V.X. da, LUZ, H.H.V. As implicações do alcoolismo na vida social e familiar do indivíduo dependente. Uniedu, 2016.

VEDANA, E.; ANSELMO, P.de M.; NEVES, J. da R. das G. e LONGO, G. Prevalência de obesidade e fatores potencialmente causais em adultos em região do sul do Brasil.. Prevalência de obesidade e fatores potencialmente causais em adultos em região do sul do Brasil, 2008.

VIEIRA, K.L.D; GOMES V.L.D.O; BORBA, M.R; e COSTA, C.F.D.S. Atendimento da população masculina em unidade básica saúde da família: motivos para a (não) procura. Esc Anna Nery, 2013, 17(1), 120-7.

\section{Como citar este artigo (Formato ABNT):}

ROCHA, Gilciéle Novais; ARAÚJO; Isadora Ferreira; NUNES, Júlia Sousa Santos. Saúde do Homem na Atenção Básica: Prevenção e Participação nos Programas. Id on Line Rev.Mult. Psic., 2018, vol.12, n.42, Supl. 1, p. 1-13. ISSN: 1981-1179.

Recebido: 29/10/2018;

Aceito: 31/10/2018 


\section{INSTRUMENTO SEMI ESTRUTURADO}

Questionário para projeto de pesquisa com o tema: SAÚDE DO HOMEM NA ATENÇÃO BÁSICA: PREVENÇÃo E PARTICIPAÇÃO NOS PROGRAMAS DE SAÚDE EM UMA UNIDADE

BÁSICA DE SAÚDE. Aluna do X semestre de enfermagem: Gilciéle Novais Rocha.

1. Idade: anos.

2. Situação Conjugal:

Solteiro [ ] Casado [ ] União Estável [ ] Viúvo [ ]

3. Escolaridade:

Analfabeto [ ] Ensino fundamental [ ]

Ensino Médio [ ] Ensino Superior [ ]

4. Trabalho formal: Sim [ ] Não [ ]

Trabalho informal Sim [ ] Não [ ]

5. Condições/situações gerais de saúde:

Faz uso de álcool: Sim [ ] Não [ ]

Fumante: Sim [ ] Não [ ]

Faz uso de outras drogas: Sim [ ] Não [ ]

Tem hipertensão Arterial: Sim [ ] Não [ ]

Tem Diabetes: Sim [ ] Não [ ]

Tem Doença Cardíaca: Sim [ ] Não [ ]

6. Pratica alguma atividade física: Sim [ ] Não [ ]

7. Motivo da consulta:

Prevenção [ ] Tratamento [ ]

8. Sabe quais são os programas de saúde que a unidade oferece?

SIM [ ] NÃO [ ]

9. Sabe informar quais ações de prevenção voltadas para a saúde do homem que os serviço de saúde deveria oferecer?

SIM [ ] NÃO [ ]

10. Enfrenta dificuldades no atendimento na unidade de saúde?

SIM [ ] NÃO [ ]

Se Sim, qual: 